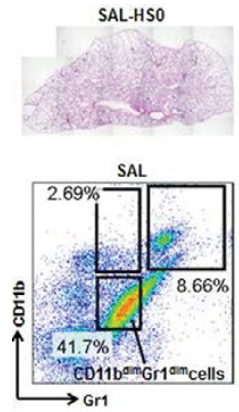

CD11 $\mathrm{b}^{\mathrm{dim}} \mathrm{Gr} 1^{\mathrm{dim}}$ cells

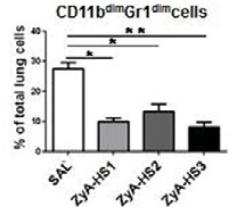

Figure 1

Disclosure of Interest: None declared

DOI: 10.1136/annrheumdis-2017-eular.1429

\section{FRI0081 A MACAQUE MODEL OF RHEUMATOID ARTHRITIS BY IMMUNIZATION WITH CITRULLINATED PEPTIDES: LESSONS FOR THE HUMAN DISEASE}

S. Bitoun ${ }^{1}$, P. Roques ${ }^{2}$, T. Larcher ${ }^{3}$, G. Nocturne ${ }^{1}$, C. Serguera ${ }^{4}$, P. Chretien ${ }^{5}$, G. Serre ${ }^{6}$, R. Le Grand ${ }^{7}$, X. Mariette ${ }^{1} .{ }^{1}$ Université Paris-Sud, Hôpitaux Universitaires Paris-Sud, INSERM U1184, le Kremlin Bicêtre; ${ }^{2}$ Immunology of viral infections and autoimmune diseases, CEA, Fontenay aux Roses: ${ }^{3}$ INRA UMR703 Veterinary School of Nantes, Nantes; ${ }^{4}$ MIRCEN, CEA/INSERM, Fontenay aux Roses: ${ }^{5}$ AP-HP, Hôpitaux Universitaires Paris-Sud, le Kremlin Bicêtre: ${ }^{6}$ INSERM U1056 - Université de Toulouse Paul, Toulouse: ${ }^{7}$ CEA Université Paris Sud 11 - INSERM U1184, Fontenay aux Roses, France

Background: Recent evolution in the understanding of rheumatoid arthritis (RA) mechanisms is the role of antibodies directed against citrullinated (cit) proteins (ACPAs). The shared epitope (SE) on the MHC class II is the main genetic risk factor of RA and favors presence of ACPAs. Mouse models dependent on cit peptides immunization require transgenic expression of the SE and are controversial. Non-human primates are ideal to study the interaction between ACPA and RA since $8 \%$ carry, similarly to humans, the SE called the $\mathrm{H} 6$ haplotype. Objectives: The goal of this study was to develop a new animal model of RA based on immunization of genetically predisposed macaques against cit peptides to generate an ACPA-mediated model of arthritis.

Methods: Six macaques were intra dermally (ID) immunized with 4 peptides: vimentin (59-71) and (66-78), $\alpha$ fibrinogen (79-91) and aggrecan (89-103). H6 animals were immunized with either cit $(n=2)$ or arginine $(\arg )(n=2)$ containing peptides. Two non $\mathrm{H} 6$ animals were immunized with cit peptides. These peptides are known to induce a $\mathrm{T}$ cell response in RA patients carrying the SE. T-cell response was assessed with Interferon $\gamma$ ELISPOT and B-cell response by ELISA. An intra articular (IA) boost was done 30 weeks after initial immunization with either incomplete Freud's adjuvant (IFA) alone, IFA and cit peptides and IFA plus non relevant peptides.

Results: In the macaques, the T-cell response was specific to cit or arg peptides (depending on the peptides used for immunization). Surprisingly, the presence of the $\mathrm{H} 6$ epitope did not influence the response. Conversely the antibodies generated in response to the peptides were cross-reactive between the cit and arg peptides. Since no clinical response was observed, an IA boost was performed with the same 4 cit peptides and IFA adjuvant. This led to a prolonged neutrophilrich mono-arthritis preferentially in $\mathrm{H} 6$ animals (Figure). Conversely, animals

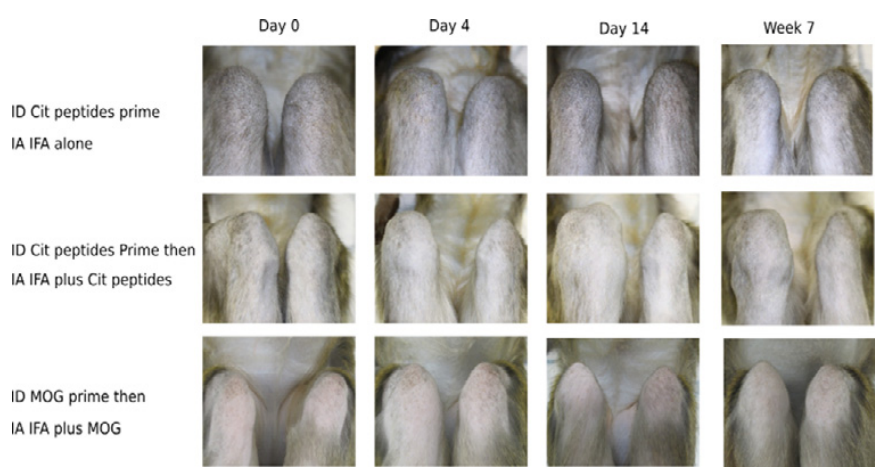

boosted with IFA alone only or with IFA plus myelin oligodendrocyte glycoprotein (MOG) peptides and previously immunized with MOG peptides presented with a transient mono-arthritis. Histological analysis revealed a local mononuclear infiltrate in one of the two animals that had prolonged knee monoarthritis. There was no clinical polyarthritis but 2 animals displayed synovial proliferation in 1 MCP and 1 MTP, respectively.

Conclusions: Immunization of macaques with cit peptides, then IA boost with the same cit peptides plus IFA, induced a prolonged monoarthritis. Shared epitope bearing did not restrict the T-cell response but seemed to favor the prolonged swelling after the IA boost. Neutrophil infiltration of the joint occurred similarly to what is seen in RA. Further use of neutrophil chemo-attractant might lead to a poly-articular disease. This macaque model of RA appears unique to study the events occurring during the pre-clinical phase of RA.

Disclosure of Interest: None declared

DOI: 10.1136/annrheumdis-2017-eular.2843

\section{FRI0082 RAS SIGNALING INHIBITORS ATTENUATE ARTHRITIS IN ANIMAL MODELS OF RHEUMATOID ARTHRITIS BY DOWN MODULATING THE PATHOGENIC TH17 CELL RESPONSE}

M. Zayoud ${ }^{1,2}$, E. Vax ${ }^{1}$, G. Elad Sfadia ${ }^{3}$, V. Marcu-Malina ${ }^{1}$, Y. Kloog ${ }^{3}$, I. Goldstein 1,2,4 . ${ }^{1}$ Immunology Core Laboratory, Chaim Sheba Academic Medical Center, Ramat Gan; ${ }^{2}$ Medicine, Sackler Faculty of Medicine, Tel Aviv University; ${ }^{3}$ Faculty of Life Sciences, Tel Aviv University, Tel Aviv; ${ }^{4}$ Rheumatology, Chaim Sheba Academic Medical Center, Ramat Gan, Israel

Background: Ras-GTPases are vital for normal T cell activation, and downstream effectors of Ras include the MEK/ERK, PI3-kinase/AKT, mTOR/p70S6-kinase, and NF-kB pathways. Somatic mutations in NRAS cause an autoimmune lymphoproliferative disorder and $T$ cells from Rheumatoid Arthritis (RA) patients exhibit perturbation of the Ras/MEK/ERK pathway. The small molecule Farnesylthiosalicylic acid (FTS) inhibits the interaction between Ras-GTPases and prenyl-binding chaperones vital for proper plasma membrane localization and downstream signaling [1]. Previous pre-clinical studies suggest that FTS has an immunomodulatory effect in various animal models of autoimmunity [2].

Objectives: To test in the Lewis rat adjuvant induced arthritis (AIA) and in the $\mathrm{DBA} / 1$ mouse collagen type-II induced arthritis (CIA) models the therapeutic immunomodulatory effect of FTS alone or combined with methotrexate (MTX).

Methods: Arthritis was induced in 8-12 week old male Lewis rats by complete Freund's adjuvant (CFA) injection and in male DBA/1 mice by collagen type-II (CII) immunization. Animals were treated prophylactically with once daily oral FTS (100 $\mathrm{mg} / \mathrm{kg})$; weekly i.p injection of MTX $(0.5 \mathrm{mg} / \mathrm{kg})$, oral FTS combined with MTX, or daily oral vehicle solution ( $0.5 \%$ carboxy methyl cellulose; CMC). Arthritis severity was scored daily from disease onset until study termination. In addition, we measured multiple disease- and drug-related immunological/molecular biomarkers.

Results: AIA severity was significantly reduced by FTS treatment compared to $\mathrm{CMC}$ controls (Figure $1 \mathrm{~A}, P<0.001$ ). Combining FTS and low dose MTX significantly increased its therapeutic efficacy compared to each drug alone (Figure $1 \mathrm{~A}, P<0.05)$. FTS or FTS+MTX treatment also suppressed the upsurge in serum IL-17 and CRP compared to ailing controls. Global gene expression analysis of relevant splenic CD4+ T cells revealed that FTS is a potent inhibitor of pro-inflammatory and $\mathrm{TH} 17$ related gene networks. Next, our data from the mouse CIA model show that the therapeutic efficacy of FTS was non-inferior to MTX and it significantly reduced arthritis severity compared to controls (Figure $2, P<0.001)$. Importantly, FTS significantly inhibited the production of pathogenic anti-CII autoantibodies and upregulation of serum IL-6 and IL-17A compared to control arthritic mice. The in depth, multiplex, analysis of the effect of FTS on the $\mathrm{T}$ cell cytokine response to CII, revealed strong suppression of IL-22, IL-17, IL-9, GM-CSF and TNF production. Noteworthy, FTS therapy positively correlated with reduced Ras-GTP, p-ERK and p-AKT levels in splenic lymphocytes (drug related biomarkers).
A.

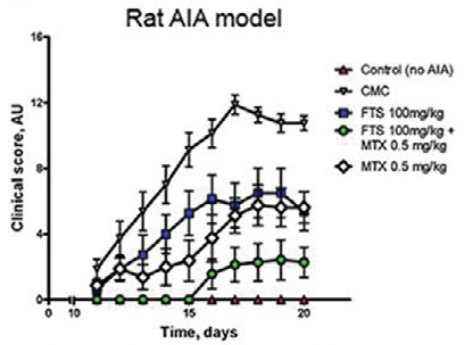

B.

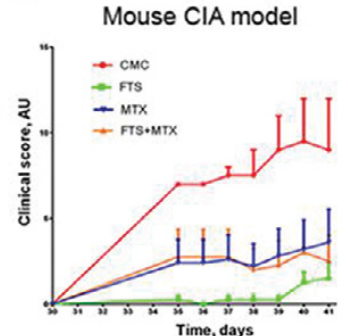

Figure 1. FTS attenuates arthritis severity in the $(A)$ rat $A I A$ and $(B)$ mouse CIA models

Conclusions: FTS, a first-in-class oral selective Ras-GTPases inhibitor, exhibits a potent immunomodulatory effect in two classical murine model of arthritis, coupled with the inhibition of the $\mathrm{TH} 17$ response to relevant arthritogenic-antigens. Thus, Ras-signaling-blockade is a promising novel therapeutic approach for RA. References:

[1] Kloog Y, Cox AD. Prenyl-binding domains: potential targets for Ras inhibitors and anti-cancer drugs. Semin Cancer Biol. 2004 Aug; 14(4):253-261. 